\title{
ON THE NON-EXISTENCE OF A CODIMENSION ONE HOLOMORPHIC FOLIATION TRANSVERSE TO A SPHERE
}

\author{
Toshikazu Ito And Bruno ScÁrdua \\ Dedicated to the memory of Professor Haruo Kitahara
}

\section{Introduction}

In this paper we address the following question:

Question 1. Is there any codimension one holomorphic foliation $\mathcal{F}$ in a neighborhood of the closed unit ball $B[0 ; 1] \subset \mathbb{C}^{n}$ such that $\mathcal{F}$ is transverse to the boundary sphere $S^{2 n-1}(0 ; 1)$ for $n \geq 3$ ?

We point-out that for $n=2$ there are linear examples and the situation is wellunderstood $([1],[5])$. We conjecture that, for dimension $n \geq 3$, Question 1 has a negative answer. In this direction we state our main result as:

Theorem 1. Let $\mathcal{F}$ be a codimension one foliation in a neighborhood $U$ of the closed unit ball $B[0 ; 1] \subset \mathbb{C}^{n}, n \geq 2$ and transverse to the boundary sphere $S^{2 n-1}(0 ; 1)$. If $\mathcal{F}$ has some leaf $L_{0}$ with $0 \in \bar{L}_{0}$ and which is closed in $U \backslash \operatorname{sing}(\mathcal{F})$ and transverse to every sphere $S^{2 n-1}(0 ; R), 0<R \leq 1$ then $n=2$.

A natural situation happens when $\mathcal{F}$ has a global separatrix: according to [7] if a codimension one foliation $\mathcal{F}$ as above is transverse to $S^{2 n-1}(0 ; 1)$ then $\mathcal{F}$ has a single singularity $p_{0}$ in the open ball $B^{2 n}(0 ; 1)$. If $n \geq 3$ then by Malgrange's Theorem $([8])$ the foliation $\mathcal{F}$ admits a local holomorphic first integral $f: V \rightarrow \mathbb{C}$ in a neighborhood $V$ of $p_{0}$ in $\mathbb{C}^{n}$ with $f\left(p_{0}\right)=0$. The germ of hypersurface $\Lambda=f^{-1}(0) \subset V$ is called a separatrix of $\mathcal{F}$, the existence of a separatrix for dimension 2 is proved in [3]. We shall say that $\mathcal{F}$ has a global separatrix $\tilde{\Lambda}$ if the leaf $L_{0}$ of $\mathcal{F}$ that contains $\Lambda \backslash\left\{p_{0}\right\}$, is closed in $U$ for $U \supset B(0 ; 1)$ small enough. In this case we put $\tilde{\Lambda}=L_{0} \cup \Lambda=L_{0} \cup\left\{p_{0}\right\}$. An immediate consequence of our main result is:

Corollary 1. Let $\mathcal{F}$ be a foliation as above, transverse to $S^{2 n-1}(0 ; 1)$ and admitting a global separatrix $\tilde{\Lambda}$ transverse to $S^{2 n-1}(0 ; R), \forall R \in(0,1]$. Then $n=2$.

A holomorphic function $f: U \rightarrow \mathbb{C}$ defined in an open subset $0 \in U \subset \mathbb{C}^{n}$ is quasi-homogeneous if there exists a holomorphic vector field $\vec{\xi}=\sum_{j=1}^{n} \alpha_{j} z_{j} \frac{\partial}{\partial z_{j}}$, with $0 \leq \alpha_{j} \in \mathbb{Q}, \forall j$, such that $d f(\vec{\xi})=\alpha \cdot f$ for some constant $\alpha \in \mathbb{C}$. A

Received by the editors May 6, 2005. 
codimension one analytic subset $\Lambda \subset U$ is quasi-homogeneous if $\Lambda=f^{-1}(0)$ for some quasi-homogeneous function $f$ as above. As a particular case of the above results we have

Corollary 2. Let $\mathcal{F}$ be a holomorphic foliation of codimension one on a neighborhood $U$ of the closed ball $B[0 ; 1] \subset \mathbb{C}^{n}, n \geq 2$. Suppose that $\mathcal{F}$ is transverse to the boundary sphere $S^{2 n-1}(0 ; 1)$ and has a quasi-homogeneous invariant hypersurface $\Lambda \subset U$. Then $n=2$.

Essentially, we reduce Question 1 of transversality for foliations to a question of transversality for a closed leaf of the foliation. Another interesting consequence we obtain is:

Corollary 3. Let $\omega$ be a closed meromorphic one-form in a neighborhood $U$ of $B[0 ; 1] \subset \mathbb{C}^{n}, n \geq 2$ and such that the corresponding holomorphic foliation $\mathcal{F}_{\omega}$ is transverse to $S^{2 n-1}(0 ; 1)$. Suppose that the polar set of $\omega$ is transverse to $S^{2 n-1}(0 ; R), \forall R \in(0,1]$. Then $n=2$. Moreover, there is a holomorphic mapping $\Phi$ from a neighborhood of $B[0 ; 1]$ to a neighborhood of the origin $0 \in \mathbb{C}^{2}$ such that either $\mathcal{F}=\Phi^{*}\left(\mathcal{L}_{\lambda}\right)$ or $\mathcal{F}=\Phi^{*}\left(\mathcal{L}_{a, m}\right)$ where $\mathcal{L}_{\lambda}$ is the linear foliation given by $x d y-\lambda y d x=0, \lambda \in \mathbb{C} \backslash \mathbb{R}_{-}$and $\mathcal{L}_{a, m}$ is the Poncaré-Dulac normal form foliation given by $x d y-\left(m y+a x^{m}\right) d x=0, \quad m \in \mathbb{N} \backslash\{0,1\}, \quad a \in \mathbb{C} \backslash\{0\}$.

\section{Preliminaries}

Let $f: U \rightarrow \mathbb{C}$ be a holomorphic function defined in a neighborhood $U$ of the ball $B[0 ; 1] \subset \mathbb{C}^{n}$ with $f(0)=0$. We fix the standard metric on $\mathbb{C}^{n}$ corresponding to the norm $\|z\|^{2}=\sum_{j=1}^{n}\left|z_{j}\right|^{2}=\sum_{j=1}^{n} z_{j} \cdot \bar{z}_{j}$ where $z=\left(z_{1}, \ldots, z_{n}\right)$. We assume that either $f$ is a submersion at each point of $f^{-1}(0)$ or that the origin is the only singularity of $f$ in $f^{-1}(0)$ and this singularity is isolated. According to Milnor [9] we have the following: For any $\varepsilon>0$ small enough $f^{-1}(0)$ is (smooth and) transverse to the sphere $S^{2 n-1}(0 ; \varepsilon)$ and the topology of the link $K(f ; R)=$ $f^{-1}(0) \cap S^{2 n-1}(0 ; R)$ is the same for all $R \in(0, \varepsilon]$. We shall use the following remark:

Lemma 1. Let $f: U \rightarrow \mathbb{C}$ be a holomorphic function as above with $f(0)=0$ and $U$ a neighborhood of $B[0 ; 1]$ in $\mathbb{C}^{n}$. Assume that $f^{-1}(0)-\{0\}$ is transverse to the spheres $S^{2 n-1}(0 ; R)$ for every $R \in(0,1]$. Then the links $K(f ; R)$ and $K(f ; 1)$ are diffeomorphic for every $R \in(0,1]$.

Proof: Denote by $\rho: \mathbb{C}^{n} \simeq \mathbb{R}^{2 n} \rightarrow[0,+\infty)$ the $C^{\infty}$-function $\rho\left(z_{1}, \ldots, z_{n}\right)=$ $\sum_{j=1}^{n}\left|z_{j}\right|^{2}$. Then we may consider the smooth manifold $M:=f^{-1}(0)-\{0\} \subset$ $U$ with its natural $C^{\infty}$ differentiable structure. Denote by $\varphi: M \rightarrow(0,+\infty)$ the restriction $\left.\rho\right|_{M}$. Then $\varphi$ is of class $C^{\infty}$ and we have $\varphi^{-1}(R)=K(f ; R)$ for every $R \in(0,1]$. Moreover an easy computation shows that a point $p \in$ $M$ is a critical point of $\varphi$ if and only if $T_{p}(M) \subset d \rho(p)^{-1}(0)$ if and only if 
$T_{p}(M) \subset T_{p}\left(S^{2 n-1}(0 ;\|p\|)\right)$. Since by hypothesis $M$ is transverse to every sphere $S^{2 n-1}(0 ; R)$ it follows that $\varphi$ has no critical points on $M$. Now clearly $\varphi$ is proper in $M \backslash[B[0 ; \varepsilon] \cap M]$ for every $\varepsilon>0$. Therefore by standard arguments of Morse Theory the flow of the gradient of $\varphi$ gives a diffeomorphism from $K(f ; 1)$ with $K(f ; R)$ for every $0<\varepsilon \leq R<1$.

Now we investigate some examples of the situation in Lemma 1. Let $f: U \rightarrow \mathbb{C}$ be a quasi-homogeneous holomorphic function with respect to the vector field $\vec{\xi}=\sum_{j=1}^{n} \alpha_{j} z_{j} \frac{\partial}{\partial z_{j}}$ as in the introduction. In this case $d f(\vec{\xi})$ vanishes identically on $\{f=0\}$ and therefore $f^{-1}(0)$ is a union of orbits of $\vec{\xi}$. On the other hand the hermitian product of $\vec{\xi}$ with the radial vector field $\overrightarrow{\mathcal{R}}=\sum_{j=1}^{n} z_{j} \frac{\partial}{\partial z_{j}}$ is $\langle\vec{\xi}, \overrightarrow{\mathcal{R}}\rangle=\sum_{j=1}^{n} \alpha_{j}\left|z_{j}\right|^{2} \geq 0$. This product vanishes only at the origin and therefore $\vec{\xi}$ is transverse to the spheres $S^{2 n-1}(0 ; R), \forall R \in(0,1]$, showing that $f^{-1}(0)$ is transverse to $S^{2 n-1}(0 ; R), \forall R>0$ provided that the origin is an isolated singularity of $f$. For instance we can take $f=\sum_{j=1}^{n} z_{j}^{m_{j}}, \quad m_{j} \in \mathbb{N}$ and $\vec{\xi}=\sum_{j=1}^{n} \frac{1}{m_{j}} z_{j} \frac{\partial}{\partial z_{j}} \cdot$ Since we only ask for the transversality of the level $f^{-1}(0)$

with the spheres $S^{2 n-1}(0 ; R), 0<R \leq 1$ we may obtain other examples of functions $f$ with $f^{-1}(0)-\{0\}$ transverse to small spheres centered at the origin, by considering functions of the form $f=f_{0}+P$, where $f_{0}$ is quasi-homogeneous and $P$ is a small perturbation.

Lemma 2. If $\Lambda \subset U$ is quasi-homogeneous and has an isolated singularity at the origin then $\Lambda$ is transverse to the spheres $S^{2 n-1}(0 ; R), \forall R>0$.

\section{Proof of the results}

Let $\mathcal{F}$ be a holomorphic foliation of codimension one in $U ; B[0 ; 1] \subset U \subset \mathbb{C}^{n}$, $n \geq 2$ and transverse to $S^{2 n-1}(0 ; 1)$. We may assume that $n \geq 3$. According to [7] we must have $n$ even and $\operatorname{sing}(\mathcal{F}) \cap B(0 ; 1)=\left\{p_{0}\right\}$ is a single simplesingularity. In particular $n \geq 4$. We can assume that either $\mathcal{F}$ has a global separatrix in the situation of Corollary 1 or, more generally, that $\mathcal{F}$ has a closed leaf $L_{0}$ in $U \backslash \operatorname{sing}(\mathcal{F})=U \backslash\left\{p_{0}\right\}$ with $0 \in \bar{L}_{0}$. By Remmert-Stein Theorem [10] the closure $\bar{L}_{0} \subseteq L_{0} \cup\left\{p_{0}\right\}$ is an analytic subvariety of $U$, of pure codimension one and, since $U$ is a neighborhood of $B[0 ; 1]$, by a classical Theorem of Cartan, there exists a holomorphic function $f: U \rightarrow \mathbb{C}$ such that $f(0)=0$ and $\bar{L}_{0}=$ $f^{-1}(0)$. Now, according to Milnor since $f$ has an isolated singularity at the origin (or even $f$ is non-singular) and $n \geq 4$, the link $f^{-1}(0) \cap S^{2 n-1}(0 ; \varepsilon)=$ $K(f, \varepsilon)$ is simply-connected $([4],[9])$ for any $\varepsilon>0$ small enough. Lemma 1 then implies that the link $K(f ; 1)$ is also simply-connected. Let us use the 
transversality $\mathcal{F} \pitchfork S^{2 n-1}(0 ; 1)$. Denote by $\mathcal{F}_{1}$ the restriction $\left.\mathcal{F}\right|_{S^{2 n-1}(0 ; 1)}$ then $\mathcal{F}_{1}$ is a codimension two real foliation with a natural transversely holomorphic structure. Also the link $K(f, 1)$ corresponds to a simply-connected compact leaf of $\mathcal{F}_{1}$. From now on we proceed as in [6] in order to obtain a contradiction. First we apply the Global Stability Theorem of [2] to conclude that every leaf of $\mathcal{F}_{1}$ is compact with trivial fundamental group. This implies that the leaf space $S^{2 n-1}(0 ; 1) / \mathcal{F}_{1}$ of $\mathcal{F}_{1}$ is a compact Riemann surface and admits therefore some non-constant meromorphic mapping $S^{2 n-1}(0 ; 1) / \mathcal{F}_{1} \rightarrow \overline{\mathbb{C}}=\mathbb{C} \cup\{\infty\}$ onto the Riemann Sphere (it is possible to prove directly that $S^{2 n-1}(0 ; 1) / \mathcal{F}_{1}$ is simplyconnected and therefore isomorphic to $\overline{\mathbb{C}}$ ). Using this we obtain a transversely holomorphic first integral $F_{1}: S^{2 n-1}(0 ; 1) \rightarrow \overline{\mathbb{C}}$ for $\mathcal{F}_{1}$. By transversality of $\mathcal{F}$ with $S^{2 n-1}(0 ; 1)$ the map $F_{1}$ admits an extension to a holomorphic map $F: W \rightarrow \overline{\mathbb{C}}$ in a neighborhood $W_{0}$ of $S^{2 n-1}(0 ; 1)$ in $U$ and constant along the leaves of the restriction $\left.\mathcal{F}\right|_{W_{0}}$. By standard Hartogs' Extension results we can extend $F$ as a holomorphic mapping $F: W_{0} \cup B[0 ; 1] \rightarrow \overline{\mathbb{C}}$ constant along the leaves of $\mathcal{F}$. By Stein's Factorization Theorem we may assume that $F$ has connected fibers. We may write $F=\frac{\alpha}{\beta}$ for some holomorphic functions $\alpha$, $\beta$ in a neighborhood $W$ of $B[0 ; 1]$ and without non-trivial common factors in $\mathcal{O}(W)$. Since the only singularity of $\mathcal{F}$ in $B(0 ; 1)$ admits a local first integral of holomorphic type into $\mathbb{C}$ it follows that $\alpha^{-1}(0) \cap \beta^{-1}(0)=\emptyset$ and $F$ has no indeterminacy points in $B[0 ; 1]$. In particular the restriction $F_{1}=\left.F\right|_{S^{2 n-1}(0 ; 1)}$ defines a locally trivial $C^{\infty}$ fibration of $S^{2 n-1}(0 ; 1)$ over the sphere $S^{2} \simeq \mathbb{C} \cup\{\infty\}$ with simply-connected fibers. By the homotopy sequence of a fibration [11] we must have $2 n-1 \leq 3$, contradiction. This proves Theorem 1 .

Proof of Corollary 1. Let $\omega$ be a closed meromorphic one form in $U \supset B(0 ; 1)$. Write $(\omega)_{\infty}=\bigcup_{j=1}^{r}\left\{f_{j}=0\right\}$ for suitable (reduced) holomorphic functions $f_{j}: U \rightarrow$ $\mathbb{C}, j=1, \ldots, r$. Since we can take $U$ simply-connected $\omega$ can be written

$$
(*) \omega=\sum_{j=1}^{r} \lambda_{j}\left(d f_{j} / f_{j}\right)+d\left(g / \prod_{j=1}^{r} f_{j}^{n_{j}-1}\right)
$$

for some $\lambda_{j} \in \mathbb{C}$ and $n_{j} \in \mathbb{N}$ and some holomorphic function $g: U \rightarrow \mathbb{C}$. In particular either $\omega$ is holomorphic in $U$ or $\left\{f_{1}=0\right\} \subset(\omega)_{\infty}$ gives a closed leaf of $\mathcal{F}_{\omega}$. In this last case we apply Theorem 1 to obtain $n=2$. In the first case $\omega=$ $d g$ and $\mathcal{F}_{\omega}$ admits a holomorphic first integral in $U$. By the Maximum modulus principle we conclude that $\mathcal{F}_{\omega}$ cannot be transverse to $S^{2 n-1}(0 ; 1)$ even for $n=2$. Thus $n=2$ and we have a unique simple singularity for $\mathcal{F}_{\omega}$ in the ball $B(0 ; 1)$ which is necessarily a singularity in the Poincaré-domain (cf. [5]). By PoincaréDulac theorem we know that either $\mathcal{F}_{\omega}$ is linearizable as $\mathcal{L}_{\lambda}$ with $\lambda \in \mathbb{C} \backslash \mathbb{R}_{-}$in a neighborhood of the singularity or it is analytically conjugate in a neighborhood of the singularity to a Poincaré-Dulac normal form $\mathcal{L}_{a, m}$. Comparing these local models for $\omega$ with the global writing $(*)$ above we conclude. 
Corollary 2 follows immediately from Lemma 2 and Corollary 1.

Remark 1. If we do not assume that $0 \in \bar{L}_{0}$ in Theorem 1 then we cannot apply Lemma 1 in its present formulation. Nevertheless, we can proceed as follows. Suppose that $p_{0} \neq 0$ and let $T$ be an automorphism of the closed ball $B^{2 n}[0 ; 1]$ which maps $p_{0}$ to 0 and such that $T^{2}=I d$. Then both $\mathcal{F}$ and the pull-back foliation $T^{*}(\mathcal{F})$ are transverse to the sphere $S^{2 n-1}(0 ; 1)$. The foliation $T^{*}(\mathcal{F})$ has a leaf $T^{-1}\left(L_{0}\right)$ which is closed in $T^{-1}(U) \backslash \operatorname{sing}\left(T^{*}(\mathcal{F})\right)=T^{-1}(U) \backslash\{0\}$ and transverse to all hyperbolic balls of hyperbolic center $T^{-1}(0)=p_{0}$. Lemma 1 can be stated accordingly to this situation with essentially the same proof and also the notion of quasi-homogeneity. This suggests that Theorem 1 might hold for codimension one holomorphic foliations transverse to the boundary of a strongly convex domain and having a global separatrix transverse to the boundary of all Caratheodory or Kobayashi balls centered at some singularity. We want to thank the referee for this and other valuable remarks.

\section{References}

[1] M. Brunella, On holomorphic foliations in complex surfaces transverse to a sphere, Bol. Soc. Brasil. Mat. (N.S.) 26 (1995), no. 2, 117-128.

[2] — A Global Stability Theorem for transversely holomorphic foliations, Ann. Global Anal. Geom. 15 (1997), no. 2, 179-186.

[3] C. Camacho, P. Sad, Invariant varieties through singularities of holomorphic vector fields, Ann. of Math. (2) 115 (1982), no. 3, 579-595.

[4] A. Dimca, Singularities and Topology of Hypersurfaces. Universitext, Springer Verlag, 1992.

[5] T. Ito, A. Poincaré-Bendixson type theorem for holomorphic vector fields, RIMS Pub. 878 (June 1994), 1-9.

[6] T. Ito, B. Scárdua, On holomorphic foliations transverse to spheres. To appear in Moscow Mathematical Journal, 2005.

[7] _ A Poincaré-Hopf Index Theorem for holomorphic one-forms. Topology 44 (2005) no.1, 73-84.

[8] B. Malgrange, Frobenius avec singularités [d'aprés B. Malgrange, J. F. Mattei et R. Moussu]. (French) Séminaire Bourbaki, 30e année (1977/78), Exp. No. 523, pp. 290-299, Lecture Notes in Math., 710, Springer, Berlin, 1979.

[9] J. Milnor, Singular points of complex hypersurfaces. Ann. Math. Studies, Vol. 61, Princeton Univ. Press, 1968.

[10] R. C. Gunning, Introduction to holomorphic functions of several variables. Vol. II. Local theory. The Wadsworth \& Brooks/Cole Mathematics Series. Wadsworth \& Brooks/Cole Advanced Books \& Software, Monterey, CA, 1990.

[11] N. Steenrod, The topology of fibre bundles. Reprint of the 1957 edition. Princeton University Press, Princeton, NJ, 1999.

Department of Natural Science, Ryukoku University, Fushimi-Ku, Kyoto 612-8577, JAPAN

Instituto de Matemática C.P. 68530, Universidade Federal do Rio de Janeiro, 21.945-970 RiO DE JANEIRO-RJ, BRAZIL

E-mail address: scardua@impa.br 